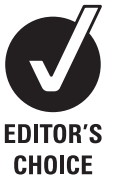

CHOICE
${ }^{1}$ Division of Cardiovascular and Neuronal Remodelling, Leeds Institute of Genetics, Health and Therapeutics, University of Leeds, Leeds, UK

${ }^{2}$ Kleijnen Systematic Reviews, Escrick Business Park, York, UK ${ }^{3}$ Department of Neuroradiology, Leeds General Infirmary, Leeds, UK

${ }^{4}$ Department of Cardiology, Leeds General Infirmary, Leeds, UK

\section{Correspondence to}

Dr JP Greenwood, Division of Cardiovascular and Neuronal Remodelling, Leeds Institute of Genetics, Health and Therapeutics, University of Leeds, Leeds, UK; j.greenwood@leeds.ac.uk

Accepted 6 June 2011 Published Online First 7 July 2011

\title{
Diffusion-weighted MRI determined cerebral embolic infarction following transcatheter aortic valve implantation: assessment of predictive risk factors and the relationship to subsequent health status
}

\author{
Timothy A Fairbairn, ${ }^{1}$ Adam N Mather, ${ }^{1}$ Petra Bijsterveld, ${ }^{1}$ Gillian Worthy, ${ }^{2}$ Stuart Currie, ${ }^{3}$ \\ Anthony J P Goddard, ${ }^{3}$ Daniel J Blackman, ${ }^{4}$ Sven Plein, ${ }^{1,4}$ John P Greenwood ${ }^{1,4}$
}

\section{ABSTRACT}

Background 'Silent' cerebral infarction and stroke are complications of transcatheter aortic valve implantation (TAVI).

Objective To assess the occurrence of cerebral infarction, identify predictive risk factors and examine the impact on patient health-related quality of life (HROoL). Methods Cerebral diffusion weighted MRI of 31 patients with aortic stenosis undergoing CoreValve TAVI was carried out. HROoL was assessed at baseline and at 30 days by SF-12v2 and E05D questionnaires.

Results New cerebral infarcts occurred in 24/31 patients $(77 \%)$ and stroke in $2(6 \%)$. Stroke was associated with a greater number and volume of cerebral infarcts. Age $(r=0.37, p=0.042)$, severity of atheroma (arch and descending aorta; $r=0.91, p<0.001, r=0.69, p=0.001$, respectively) and catheterisation time $(r=0.45, p=0.02)$ were predictors of the number of new cerebral infarcts. HROoL improved overall: SF-12v2 physical component summary increased significantly ( $32.4 \pm 6.2$ vs $36.5 \pm 7.2$; $p=0.03$ ) with no significant change in mental component summary ( $43.5 \pm 11.7$ vs $43.1 \pm 14.3 ; p=0.85)$. The E05D score and Visual Analogue Scale showed no significant change $(0.56 \pm 0.26$ vs $0.59 \pm 0.31 ; p=0.70$, and $54.2 \pm 19$ vs $58.2 \pm 24 ; p=0.43$ ).

Conclusion Multiple small cerebral infarcts occurred in $77 \%$ of patients with TAVI. The majority of infarcts were 'silent' with clinical stroke being associated with a both higher infarct number and volume. Increased age and the severity of aortic arch atheroma were independent risk factors for the development of new cerebral infarcts. Overall HROOL improved and there was no association between the number of new cerebral infarcts and altered health status.

\section{INTRODUCTION}

Transcatheter aortic valve implantation (TAVI) has rapidly developed as an effective treatment for patients with severe symptomatic aortic stenosis (AS) who are not suitable for surgical aortic valve replacement. With over 20000 patients treated worldwide using either the Edwards SAPIEN (Edwards Lifesciences, Irvine, California, USA) or CoreValve (Medtronic, Minneapolis, Minnesota, USA), it reflects the increasing prevalence of AS in an ageing population and observations that $30 \%$ of individuals with severe AS are not offered surgery. ${ }^{1}$ TAVI outcome data have demonstrated better clinical outcomes than standard medical treatment, $97 \%$ procedural success, 6.4-8\% 30 day and 22-31\% 1 year allcause mortality. ${ }^{2-4}$ Stroke is an important complication that occurs in $0.6-5 \%$ of patients with TAVI compared with $1.1 \%$ of patients treated with standard medical treatment. ${ }^{4}$ It is believed to be secondary to ischaemic embolic events, either from aortic atheroma during the passage of a delivery catheter or degenerative valvular material released during the valvuloplasty and valve deployment. Procedural cerebral infarction can occur more frequently than is clinically apparent. ${ }^{6-8}$ These 'silent' ischaemic events have been documented after cardiac surgery, ${ }^{9} 10$ and are associated with neurological dysfunction and future cognitive decline. ${ }^{11}$ They may have a substantial effect upon an individual's quality of life, potentially affecting patient selection and treatment recommendations, particularly if TAVI is expanded to a larger, younger, lower-risk patient group.

The aims of our study, therefore, were to identify the demographic and procedural risk factors associated with new cerebral infarction and to assess the impact of these new lesions upon patient's subsequent health status.

\section{METHODS}

Between May 2008 and August 2010, 85 patients underwent TAVI at our institution. Each person was assessed by a multidisciplinary team (cardiothoracic surgeon, cardiologist, cardiac anaesthetist) in accordance with international guidelines. ${ }^{12}$ Severe AS was defined as an aortic valve area of $<0.8 \mathrm{~cm}^{2}$ or a peak velocity of $>4 \mathrm{~m} / \mathrm{s}$ on echocardiography. All patients were $\geq 65$ years of age, and had a logistic EuroSCORE $>20$, or comorbidities that precluded cardiac surgery. Exclusion criteria for the study were MRI-incompatible factors including pacemaker, claustrophobia or an inability to lie flat. The study complied with the declaration of Helsinki, was approved by the local research ethics committee and all patients provided written informed consent.

\section{Transcatheter aortic valve implantation}

Patients were screened using echocardiography, invasive coronary angiography and aortography. Data collected included patient demographics, comorbidity and potential risk factors for 
cerebrovascular accident. Ejection fraction, left ventricular enddiastolic dimensions and aortic valve pressure gradient were recorded by transthoracic echocardiography. Aortic atheroma was assessed in the ascending, descending and arch of the aorta by transoesophageal echocardiography (TOE), then graded for severity $(1-5)$ by an experienced reader according to previously published criteria. ${ }^{13}$ Aortic valve calcification was assessed by a combination of transthoracic echocardiography and TOE and graded for severity: I, no calcification; II, mild calcification; III, moderate calcification; IV, severe calcification. ${ }^{14}$ TAVI was performed in all patients using the third-generation 18-Fr Medtronic CoreValve ReValving system. A standard technique was employed for implantation of the CoreValve prosthesis as previously described..$^{2}$ All procedures were performed under general anaesthesia with TOE guidance and aortic balloon valvuloplasty before valve deployment. Patients received dual antiplatelet treatment with aspirin $75 \mathrm{mg}$ and clopidogrel $75 \mathrm{mg}$ daily, continued for a minimum of 6 months postoperatively. Heparin was administered during the procedure to maintain an activated clotting time $>200$ s.

\section{MRI}

Diffusion weighted (DW)-MRI was performed using a 1.5 Tesla system (Intera, Phillips Healthcare, Best, Netherlands or Avanto, Siemens Medical Systems, Erlangen, Germany). Pre- and postprocedure scans were performed on the same scanner using an identical imaging protocol: T2 weighted fast field echo, turbo field echo and diffusion weighted imaging (DWI). Imaging parameters were: 22 slices, $5 \mathrm{~mm}$ thick, $1 \mathrm{~mm}$ gap, FOV 350, RFOV 100. Two neuroradiologists blinded to the clinical and procedural details independently reported the scans. Lesion size $(<5 \mathrm{~mm}$ or $\geq 5 \mathrm{~mm}$ ) and volume $(\mathrm{ml})$ were quantified by planimetry using post-processing software (Omass, V7.0, Medis).

\section{Neurological and health status assessment}

Clinical examination of patients after TAVI (days 1 and 2) was performed by an experienced medical doctor to assess for neurological signs according to the National Institutes of Health Stroke Scale. Symptoms and signs of $<24 \mathrm{~h}$ duration were defined as a transient ischaemic attack and if they persisted over $24 \mathrm{~h}$ they were classified as a stroke.

No disease-specific questionnaire exists for patients with AS, therefore health status was assessed using two well-validated, generic Health-Related Quality-of-Life (HROoL) Questionnaires: SF-12v2 and EO5D. Each questionnaire was administered by a trained nurse or doctor and completed by the patient before (baseline) and at 30 days after the procedure by postal or telephone survey. The SF-12v2 Health Outcomes Questionnaire (QualityMetric, Lincoln, Rhode Island, USA) assesses HRQoL using eight dimensions each with a score of $0-100$. It is a shorter, simpler version of the well-validated SF- $36^{15}$ and is thus more suited to an elderly population. This produces (1) a physical component summary (PCS) of physical functioning, role-physical, bodily pain and general health; (2) a mental component summary (MCS) score of vitality, social functioning, role-emotional and mental health. EO5D (EuroOoL) uses five dimensions to measure HRQoL: mobility, self-care, usual activities, pain/discomfort and anxiety/depression. Scoring is from 0 (death) to 1 (full health). The second component of the EO5D is a Visual Analogue Scale (VAS) of 0-100 (worst imaginable health to best possible health).

\section{Statistical analysis}

Statistical analysis was performed using the PASW software package (V 17.0 SPSS). Continuous data are presented as mean $\pm \mathrm{SD}$, categorical data as frequencies and percentages. Test for normality was assessed using the Shapiro-Wilks goodnessof-fit test and $\mathrm{Q}-\mathrm{Q}$ plots. Continuous variables were compared using the paired $t$ test. Categorical variables were compared using the Mann-Whitney or Kruskal-Wallis tests. Linear regression analysis was used to identify the relationship between patient and procedural factors and the number of new cerebral infarctions following TAVI. Univariate analysis was used to identify individual predictors. Variables with a univariate significance of $p<0.1$ were entered into a multiple stepwise regression analysis to determine the independence of these predictors. Multivariate analysis was also performed using the number of new infarcts and stroke as two dependent variables and the univariate predictors as factors and covariates. A twosided significance level of $p<0.05$ was considered statistically significant.

\section{RESULTS}

\section{Patient population}

Forty of the 85 patients consented to recruitment to the study and underwent baseline pre-procedure DW-MRI to identify any pre-existing lesions (figure 1). Of these, 31 (78\%) completed paired head scans before (median 1 day, IOR 1-2) and after TAVI (median 5 days, IOR 4-6). Of the remaining nine patients, TAVI was not performed on four individuals, and five individuals underwent TAVI but could not complete the MRI study because three patients required permanent pacemakers after valve implantation, one patient was claustrophobic and one was unable to lie flat in the scanner. The demographic and echocardiographic details of the 31 patients (mean age $81 \pm 5.9$ years; 20 (65\%) women, table 1) are representative of a typical unselected TAVI population.

\section{Procedural data}

A $26 \mathrm{~mm}$ CoreValve was deployed in 11 (35\%) cases and a $29 \mathrm{~mm}$ CoreValve in 20 (65\%), via either the femoral $26(84 \%)$ or subclavian $5(16 \%)$ artery. Procedural success was $98 \%$, with an average catheterisation time of $68 \pm 26 \mathrm{~min}$, fluoroscopy time of $22 \pm 6 \mathrm{~min}$ and $150 \pm 47 \mathrm{ml}$ of contrast given. Valve dislocation and retrieval occurred in only two $(6 \%)$ individuals. Despite this low occurrence, this resulted in a significantly longer catheterisation and fluoroscopy time (64 \pm 22 vs $106 \pm 48, p=0.02$ and $21 \pm 5$ vs $35 \pm 9, \mathrm{p}=0.05 \mathrm{~min}$ respectively). After the procedure dilatation of the self-expanding valve was required in 11 (35\%) cases, with no significant increase in either catheterisation or

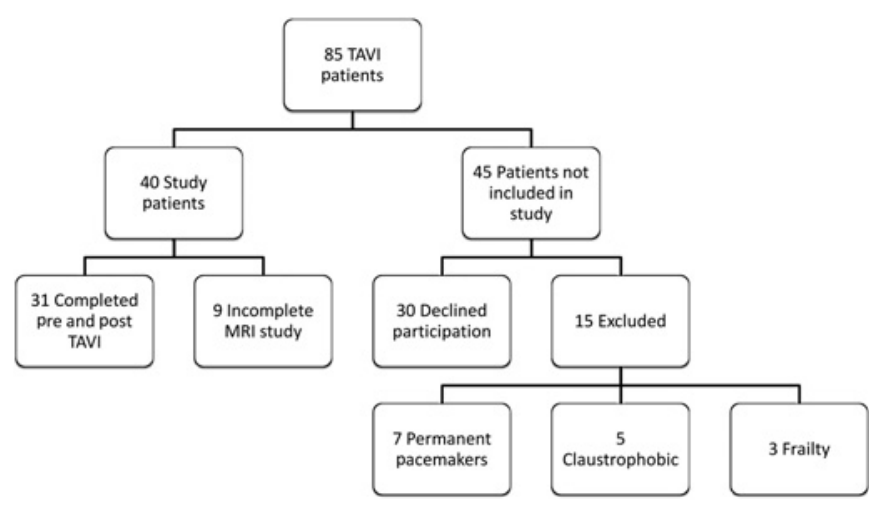

Figure 1 Patient recruitment. A flow diagram demonstrating patient recruitment, inclusion and exclusion criteria. TAVI, transcatheter aortic valve implantation. 
Table 1 Baseline patient characteristics of the study population

\begin{tabular}{|c|c|}
\hline Characteristics & Study population $(n=31)$ \\
\hline Age & $81( \pm 5.9)$ \\
\hline Female gender, $\mathrm{n}(\%)$ & $20(65)$ \\
\hline $\mathrm{BMI}\left(\mathrm{kg} / \mathrm{m}^{2}\right)$ & $27( \pm 4.3)$ \\
\hline Logistic EuroSCORE (\%) & $23.9( \pm 16.4)$ \\
\hline AV Pressure gradient $(\mathrm{mm} \mathrm{Hg}$ ) & $62.2( \pm 23.9)$ \\
\hline AV EOA $\left(\mathrm{cm}^{2}\right)$ & $0.55( \pm 0.13)$ \\
\hline \multicolumn{2}{|l|}{ LV ejection fraction, n (\%) } \\
\hline Good & $18(58)$ \\
\hline Moderate & $12(39)$ \\
\hline Poor & $1(3)$ \\
\hline LV EDD (mm) & $47( \pm 7.3)$ \\
\hline Creatinine $(\mu \mathrm{mol} / \mathrm{l})$ & 114 (IOR 90-178) \\
\hline Hypertension, n (\%) & $14(45)$ \\
\hline Hypercholesterolaemia, n (\%) & $20(65)$ \\
\hline Diabetes, n (\%) & $9(29)$ \\
\hline Atrial fibrillation, $\mathrm{n}(\%)$ & $7(23)$ \\
\hline Cerebrovascular disease, $\mathrm{n}(\%)$ & $3(10)$ \\
\hline Peripheral vascular disease, $\mathrm{n}$ (\%) & $7(23)$ \\
\hline Porcelain aorta, n (\%) & $9(29)$ \\
\hline
\end{tabular}

$\mathrm{AV}$, aortic valve; BMI, Body Mass Index; EOA, estimated orifice area; EDD, end-diastolic dimension; LV, left ventricular.

fluoroscopy times compared with those without post-dilatation $(61 \pm 15$ vs $77 \pm 37, p=0.18$ and $22 \pm 5$ vs $23 \pm 8, p=0.77 \mathrm{~min}$, respectively). TOE identified aortic valve calcification in all individuals, 20 (65\%) patients had severe aortic valve calcification, seven (22\%) moderate and four (13\%) mild. Aortic atheroma classification and grading are reported in figure 2. Increased age was significantly related to the severity of aortic atheroma $(p=0.016)$.

\section{Cerebral infarction on diffusion-weighted imaging}

New cerebral infarction occurred in 24 (77\%) patients. A total of 131 new infarcts occurred, distributed equally between the cerebral hemispheres (left 53\%, right 47\%), in multiple territories but predominantly in the regions of the following cerebral arteries: anterior $7 \%$, middle $59 \%$, posterior $14 \%$ and vertebrobasilar $20 \%$. The number of infarcts per patient was a mean of $4.2 \pm 6.5$ and median of 2 (IOR $1-5$ ), with an average infarcted tissue volume of $2.05 \pm 3.5 \mathrm{ml}$. These were mostly of a small size ( $<5 \mathrm{~mm}, \mathrm{n}=19,79 \%)$. The number of new lesions significantly correlated with the overall volume of infarct $(\mathrm{r}=0.82, \mathrm{p}<0.001)$, but interestingly not with the size of the

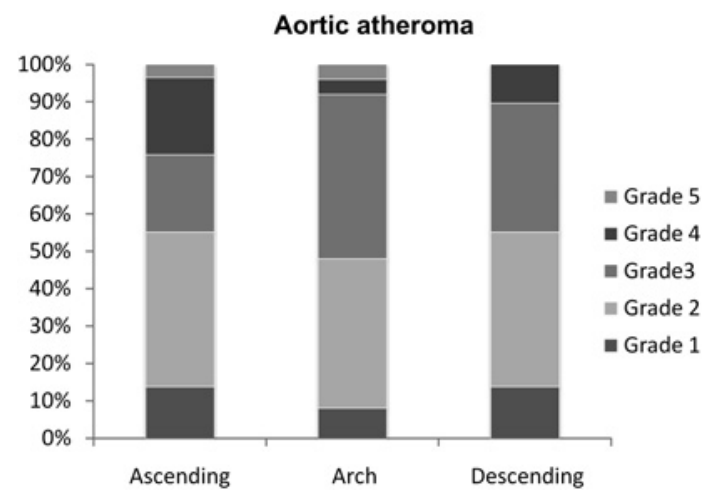

Figure 2 Aortic atheroma. Atheroma was classified in the ascending, arch or descending aorta then graded as: I, normal to mild intimal thickening; II, severe intimal thickening; III, atheroma $<5 \mathrm{~mm}$; IV, atheroma $>5 \mathrm{~mm}$; V, mobile atheroma of any size. individual infarcts $(p=0.61)$. An example of new cerebral infarction using DW-MRI is shown in figure 3.

New neurological signs were seen in two patients $(6 \%)$. The first presented with an expressive dysphasia and scored 4 on the National Institutes of Health Stroke Scale, while the second developed gait ataxia and scored 2. DWI confirmed 26 new lesions in each of these patients, distributed over several territories. These neurological deficits were diagnosed postoperatively on day 1 , persisted at day 2 ( $>24 \mathrm{~h}$ ) and were therefore classified as a stroke. When compared with those patients without neurological signs, individuals with new neurological signs demonstrated a significantly higher number $(26 \pm 0$ vs $2.7 \pm 3, \quad p=0.004)$ and volume $(11.9 \pm 6 \mathrm{ml}$ vs $1.1 \pm 1.1 \mathrm{ml}, \mathrm{p}=0.007)$ of new cerebral infarcts, although patient numbers are small.

\section{Demographic and procedural risk factor assessment}

Risk factors were assessed for a relationship to the number of new infarcts, as reported in table 2. Increased age was significantly related to a higher number of new infarcts ( $r=0.37$, $p=0.042$ ). There were no associations between the comorbidities of hypertension, hyperlipidaemia, diabetes mellitus, atrial fibrillation, cerebrovascular disease, peripheral vascular disease and a porcelain (heavily calcified) aorta, and the number of new infarcts or the development of a clinical stroke. Risk calculation using logistic EuroSCORE had no predictive relationship for the number of new cerebral infarcts $(r=-0.08, p=0.69)$ or clinical stroke. Atheroma burden in the arch and descending thoracic aorta (but not in the ascending aorta, $r=0.4, p=0.35$ ), was significantly associated with the number of new cerebral infarcts $(\mathrm{r}=0.91, \mathrm{p}<0.001 ; \mathrm{r}=0.69, \mathrm{p}=0.001)$. The severity of aortic valve calcification was not related to the number of new cerebral infarcts $(\mathrm{p}=0.33)$.

Procedural risk factor analysis showed that increased catheterisation time was associated with the number of new infarcts $(r=0.45, p=0.02)$ whereas increased fluoroscopy time did not reach statistical significance $(r=0.36, p=0.05)$. All other procedural variables, including the size of the CoreValve (26 or $29 \mathrm{~mm}, \mathrm{p}=0.19$ ), had no relationship to the number of infarcts or presence of clinical stroke. CoreValve after dilatation or dislocation and retrieval did not effect the number of new infarcts observed ( $p=0.22$ and $p=0.93$, respectively).

The univariate variables (age, fluoroscopy time, catheterisation time, arch and descending aortic atheroma) were then entered into a multiple regression model, where age and aortic arch atheroma remained independent predictors of new cerebral infarcts ( $p=0.035$ and $p=0.023$ respectively), (table 3 ). Multivariate analysis was used to determine if the univariate variables could predict the occurrence of stroke as well as the number of new infarcts. Aortic arch atheroma was the only variable to significantly predict both $(\mathrm{F}=7.16, \mathrm{p}=0.008)$.

\section{Health status}

HRQoL scores were interpreted in the context of the female and male averages for a similar aged population to those in our study; these were 36-38 (PCS), 50-51 (MCS), 0.69-0.71 (EQ5D) and 70-75 (VAS). ${ }^{16}$

The SF-12v2 results at baseline and 30 days are reported in figure 4 . The individual components that showed a significant increase from baseline were physical functioning, bodily pain and general health $(p=0.003, p=0.03$ and $p=0.003$, respectively). A comparison of the summary scores revealed that the PCS increased significantly from baseline $32.4 \pm 6.2$ to 30 days $36.5 \pm 7.2,(p=0.03)$ with no significant change in MCS from 

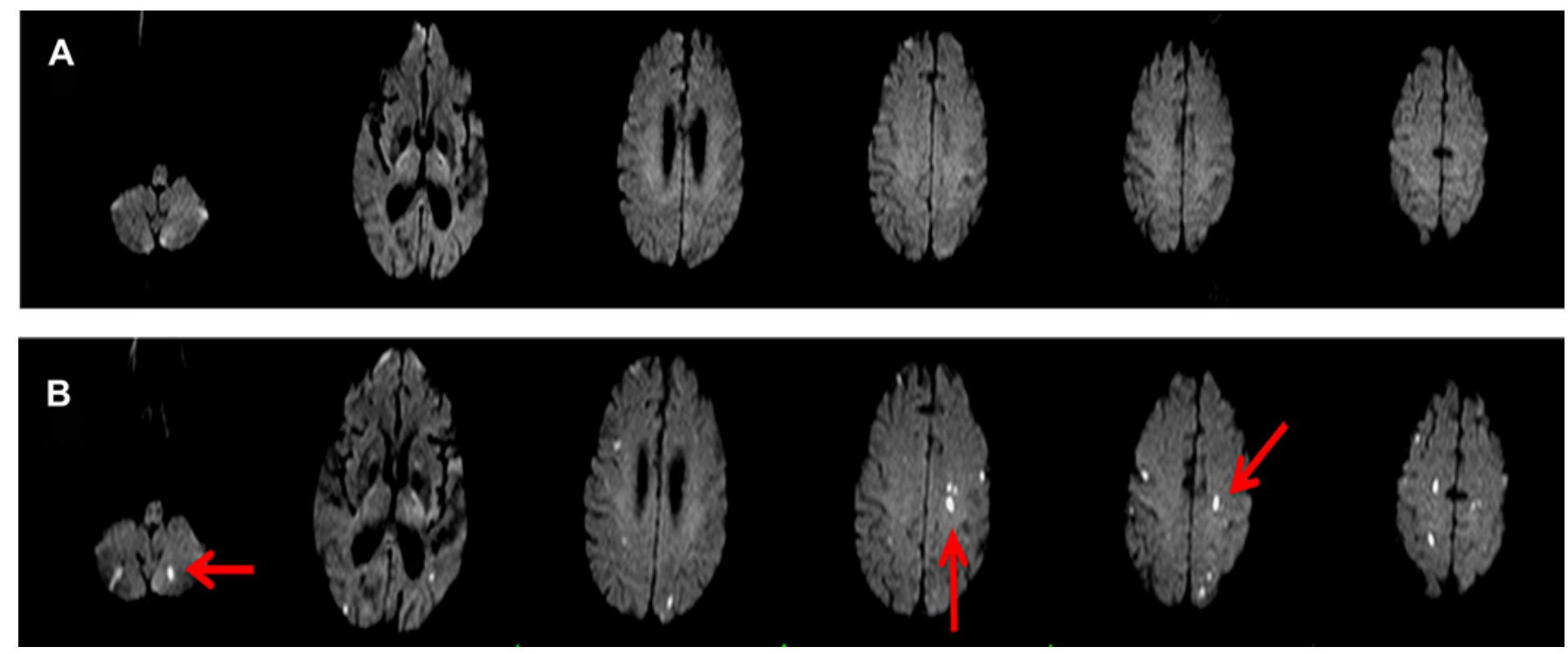

Figure 3 Diffusion weighted MRI of the brain. Cerebral images, including the cerebellum and brainstem, Before $(A)$ and after $(B)$ the transcatheter aortic valve implantation procedure. This patient had multiple new cerebral infarcts some of which are highlighted by the red arrows.

$43.5 \pm 11.7$ to $43.1 \pm 14.3$ ( $\mathrm{p}=0.85$ ). The PCS and MCS scores at 30 days showed no relationship to the number of new infarcts $(r=0.19, p=0.33 ; r=-0.08, p=0.69$, respectively), and were not significantly different between the stroke and non-stroke groups (PCS, $\mathrm{p}=0.39$ and MCS, $\mathrm{p}=0.98$ ).

The mean EQ5D score showed a non-significant increase from baseline to 30 days $(0.56 \pm 0.26$ to $0.59 \pm 0.31, p=0.70)$. Similarly, there was a non-significant increase in the VAS component of the assessment at 30 days $(54.2 \pm 19$ to $58.2 \pm 24, p=0.43)$. The EQ5D and VAS scores at 30 days showed no relationship to the number of new cerebral infarcts $(r=0.11, p=0.95 ; r=-0.085$,

Table 2 Linear regression analysis for the prediction of the number of new cerebral infarcts

\begin{tabular}{lcc}
\hline & \multicolumn{2}{l}{ Univariate analysis } \\
\cline { 2 - 3 } Risk factor & R & p Value \\
\hline Age & 0.37 & 0.042 \\
BMI $\left(\mathrm{kg} / \mathrm{m}^{2}\right.$ ) & 0.01 & 0.974 \\
EuroSCORE (Logistic) & -0.075 & 0.69 \\
AV Pressure gradient (mm Hg) & 0.11 & 0.57 \\
AV EOA (cm ${ }^{2}$ ) & -0.26 & 0.17 \\
LV ejection fraction (\%) & 0.12 & 0.52 \\
LV EDD (mm) & -0.28 & 0.14 \\
Creatinine ( $\mu$ mol/) & -0.02 & 0.90 \\
Hypertension & 0.19 & 0.30 \\
Hypercholesterolaemia & 0.11 & 0.55 \\
Diabetes & 0.1 & 0.59 \\
Atrial fibrillation & 0 & 0.93 \\
Cerebrovascular disease & 0.2 & 0.28 \\
Peripheral vascular disease & 0.06 & 0.73 \\
Porcelain aorta & 0.25 & 0.17 \\
Aortic atheroma ascending & 0.40 & 0.352 \\
Arch & 0.91 & $<0.001$ \\
Descending & 0.69 & 0.001 \\
Aortic calcification & 0.28 & 0.327 \\
Fluoroscopy time (min) & 0.36 & 0.05 \\
Catheterisation time (min) & 0.45 & 0.02 \\
Contrast dose (ml) & -0.12 & 0.53 \\
Heparin dose (U) & 0.24 & 0.19 \\
\hline AV a & & \\
\hline
\end{tabular}

\footnotetext{
$\mathrm{AV}$, aortic valve; BMI, Body Mass Index; EDD, end-diastolic dimension; EOA, estimated orifice area; LV, left ventricular.
}

$p=0.65$, respectively) and were not significantly different between the stroke and non-stroke groups $(p=0.85$ and $p=0.44$, respectively).

\section{DISCUSSION}

Previous studies have described the incidence of cerebral infarcts after TAVI but have been unable to identify any significant risk factors. ${ }^{17} 18$ This study is to our knowledge the first to demonstrate that increased age and aortic arch atheroma severity are independent predictors of the number of new cerebral infarcts following TAVI. In addition, this is the first study to assess the impact of these new cerebral infarcts on HRQoL.

The small size, high number and distribution of new cerebral infarcts suggest an embolic source. It has been demonstrated in previous cardiac catheterisation studies that embolisation can occur through the contact of a catheter with an aortic atheroma, ${ }^{19}$ and that the risk of stroke increases with the duration of procedure, fluoroscopy time and contrast dose. ${ }^{6} 7$ Our findings that prolonged catheterisation and fluoroscopy time are univariate predictors of infarct number support this as a mechanism of action in patients with TAVI, reflecting the increased contact of a catheter or valve apparatus against the aortic wall and atheroma while moving around the aortic arch.

An important finding is that the number of new cerebral infarcts is related to the severity of aortic atheroma. Evidence from non-TAVI populations has shown that atheroma plaques $>4 \mathrm{~mm}$ in the aortic arch are associated with an increased risk of stroke and death. ${ }^{20}{ }^{21}$ In this TAVI study the presence of atheroma $>5 \mathrm{~mm}$ thickness or mobile atheroma in the arch and descending

Table 3 Multiple regression analysis, univariate variables with a $\mathrm{p}$ value $<0.1$ were included in the model

\begin{tabular}{llll}
\hline \multirow{2}{*}{ Univariate predictor } & \multicolumn{3}{l}{ Multivariable analysis } \\
\cline { 2 - 4 } & B & t & p Value \\
\hline Age & 0.50 & 2.2 & 0.035 \\
Fluoroscopy time (min) & 0.23 & 1.1 & 0.27 \\
Catheterisation time (min) & 0.10 & 0.5 & 0.61 \\
Aortic arch atheroma & 3.97 & 2.4 & 0.02 \\
Descending aortic atheroma & 0.33 & 1.7 & 0.11 \\
\hline
\end{tabular}


Figure 4 Health-related quality of life-SF12. (A) Population mean scores (and SE) at baseline (black) and 30 days (light grey). Physical health: physical functioning (PF), role-physical (RP), bodily pain (BP), general health $(\mathrm{GH})$. Mental health: vitality (VT), social functioning (SF), role-emotional (RE), mental health $(\mathrm{MH}) .{ }^{*} \mathrm{p}<0.05$. (B) The physical (PCS) and mental (MCS) and 30 days. ${ }^{*} \mathrm{p}=0.03$. component summary scores at baseline

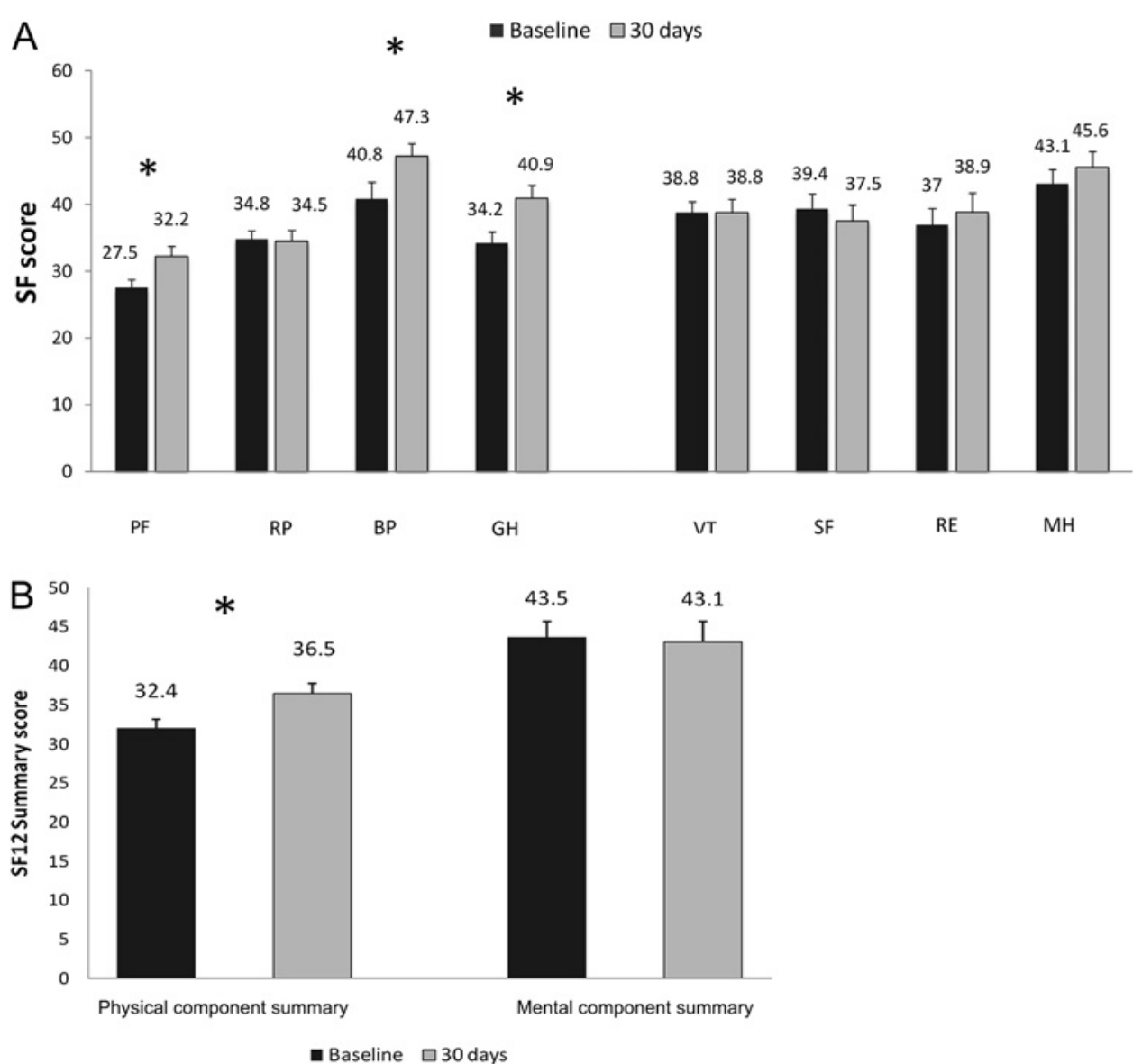

thoracic aorta was significantly associated with greater infarct number and clinical stroke. The association of descending thoracic aortic atheroma with new cerebral infarcts probably reflects its relationship with the severity of arch atheroma rather than as an independent causative factor, as atheroma increases in thickness progressively from ascending to descending aorta. ${ }^{22}$ The relationship of atheroma severity to infarct number could potentially be explained by an association with age as atherosclerotic plaques increase in thickness and complexity with advanced age. However, in this study age and aortic arch atheroma remained independent predictors of infarct number following multivariate analysis.

Our results did not identify any relationship to support the theory that embolisation occurs during balloon valvuloplasty or valve implantation. Conventional stroke risk factors such as atrial fibrillation or prior cerebrovascular disease and surgical risk (logistic EuroSCORE) were not significant predictors of new cerebral infarction or clinical stroke.

New cerebral infarcts occurred in $77 \%$ of our cohort, yet only $6 \%$ developed clinical stroke. The multiple infarcts demonstrated in these two individuals were all small ( $<5 \mathrm{~mm}$ ) except for one lesion whose anatomical distribution would not have accounted for the neurological presentation. Recent studies have demonstrated similar rates of DWI-determined cerebral infarction and clinical symptoms. ${ }^{17} 18$ The clinical significance of 'silent' infarcts remains uncertain, as concern exists about higher cognitive and neuropsychological changes such as memory, mood and personality. New cerebral infarcts have been reported in 30\% of individuals after coronary artery bypass grafting ${ }^{9}$ and $40 \%$ after surgical aortic valve replacement ${ }^{10}$; these were 'silent' in $67 \%$ of cases. These cerebral infarcts have been linked to postoperative cognitive decline (POCD), ${ }^{23}$ as well as possible dementia. ${ }^{25}$
HRQoL is an important method of assessing patient outcomes and is particularly relevant for patients with TAVI, as older age and multiple comorbidities make long-term prognostic benefit less significant. ${ }^{26}$ The SF-12v2 MCS looks at mood, emotional well-being and social functioning, all of which could be affected by POCD. Importantly, overall health status improved and the reported mental health of our patients was not affected by the number of cerebral infarcts. While an improvement in HRQoL after TAVI has been reported previously at 30 days, ${ }^{27} 3$ months ${ }^{28}$ and 5 months, ${ }^{29}$ this is the first study to assess the impact of new cerebral infarction on HRQoL in a TAVI population.

\section{Limitations} comparison group. It is difficult, however, to recruit comparable surgical patients, given that patients with TAVI by their nature are older with greater comorbidity and high surgical risk. No complex neurocognitive testing was undertaken and the health status follow-up was only up to 30 days. Our institution uses only the CoreValve for TAVI and thus did not assess the Edwards-Sapien valve or the transapical route. Interpretation of the findings related to stroke are limited by the small number of patients presenting with clinical signs. Larger studies are warranted in the future with appropriate cognitive assessment and long-term follow-up, to fully inform in a patient-specific manner the associated risks of the TAVI procedure.

\section{CONCLUSIONS}

This study found that increased age and aortic arch atheroma independently predict the number of cerebral infarctions following TAVI, irrespective of patient comorbidities or
This was a relatively small patient cohort with no direct surgical 
calculated surgical risk. The presentation of neurological signs was associated with an increased number and volume of cerebral infarcts. Aortic arch atheroma severity was the only predictor of both infarct number and stroke. Health status and quality of life improved at 30 days after TAVI with no functional mental decline. Although TAVI outcome data demonstrate better clinical outcomes than standard medical treatment, stroke remains a significant problem. ${ }^{4}$ The frequent and dispersed nature of cerebral infarcts following TAVI does suggest an embolic process, for which protection devices are already being tested. ${ }^{30}$ The identification of individuals at high risk of multiple cerebral emboli is important as it may help patient selection for the use of a protection device against stroke and POCD.

Competing interests DJB is a proctor for Medtronic CoreValve.

Ethics approval Leeds West Ethics Committee.

Contributors Authorship: Individual contributions include: TAF - design, analysis and interpretation of data, drafting and revision of manuscript. ANM, GW, SC, AJPG analysis and interpretation of data and revision of manuscript. PB - design and collection of data and revision of manuscript. DJB, SP - conception, design, collection and interpretation of data; revision of manuscript. JPG - conception, design, collection and interpretation of data; drafting and revision of manuscript.

Provenance and peer review Not commissioned; externally peer reviewed.

\section{REFERENCES}

1. lung B, Cachier A, Baron G, et al. Decision-making in elderly patients with severe aortic stenosis: why are so many denied surgery? Eur Heart J 2005;26:2714-20.

2. Piazza N, Grube E, Gerckens U, et al. Procedural and 30-day outcomes following transcatheter aortic valve implantation using the third generation (18 Fr) corevalve revalving system: results from the multicentre, expanded evaluation registry 1-year following CE mark approval. Eurolntervention 2008;4:242-9.

3. Rodés-Cabau J, Webb JG, Cheung A, et al. Transcatheter aortic valve implantation for the treatment of severe symptomatic aortic stenosis in patients at very high or prohibitive surgical risk: acute and late outcomes of the multicenter Canadian experience. J Am Coll Cardiol 2010;55:1080-90.

4. Leon MB, Smith CR, Mack M, et al. Transcatheter aortic-valve implantation for aortic stenosis in patients who cannot undergo surgery. N Engl J Med 2010;363:1597-607.

5. Zajarias A, Cribier AG. Outcomes and safety of percutaneous aortic valve replacement. J Am Coll Cardiol 2009;53:1829-36.

6. Büsing KA, Schulte-Sasse C, Flüchter $S$, et al. Cerebral infarction: incidence and risk factors after diagnostic and interventional cardiac catheterization-prospective evaluation at diffusion-weighted MR imaging. Radiology 2005:235:177-83.

7. Lund C, Nes RB, Ugelstad TP, et al. Cerebral emboli during left heart catheterization may cause acute brain injury. Eur Heart J 2005;26:1269-75.

8. Omran H, Schmidt H, Hackenbroch M, et al. Silent and apparent cerebral embolism after retrograde catheterisation of the aortic valve in valvular stenosis: a prospective, randomised study. Lancet 2003;361:1241-6.

9. Restrepo L, Wityk RJ, Grega MA, et al. Diffusion- and perfusion-weighted magnetic resonance imaging of the brain before and after coronary artery bypass grafting surgery. Stroke 2002;33:2909-15
10. Stolz E, Gerriets T, Kluge A, et al. Diffusion-weighted magnetic resonance imaging and neurobiochemical markers after aortic valve replacement: implications for future neuroprotective trials? Stroke 2004;35:888-92.

11. Goto $\mathbf{T}$, Baba $T$, Honma $K$, et al. Magnetic resonance imaging findings and postoperative neurologic dysfunction in elderly patients undergoing coronary artery bypass grafting. Ann Thorac Surg 2001;72:137-42.

12. Vahanian A, Alfieri 0 , Al-Attar N, et al. Transcatheter valve implantation for patients with aortic stenosis: a position statement from the European Association of CardioThoracic Surgery (EACTS) and the European Society of Cardiology (ESC), in collaboration with the European Association of Percutaneous Cardiovascular Interventions (EAPCI). Eur Heart J 2008;29:1463-70.

13. Hartman GS, Yao FS, Bruefach M 3rd, et al. Severity of aortic atheromatous disease diagnosed by transesophageal echocardiography predicts stroke and other outcomes associated with coronary artery surgery: a prospective study. Anesth Analg 1996:83:701-8.

14. Rosenhek R, Binder T, Porenta G, et al. Predictors of outcome in severe, asymptomatic aortic stenosis. N Engl J Med 2000;343:611-17.

15. Ware J Jr, Kosinski M, Keller SD. A 12-Item Short-Form Health Survey: construction of scales and preliminary tests of reliability and validity. Med Care 1996; 34:220-33.

16. Hanmer J, Lawrence WF, Anderson JP, et al. Report of nationally representative values for the noninstitutionalized US adult population for 7 health-related quality-of life scores. Med Decis Making 2006;26:391-400.

17. Ghanem A, Müller A, Nähle CP, et al. Risk and fate of cerebral embolism after transfemoral aortic valve implantation: a prospective pilot study with diffusionweighted magnetic resonance imaging. J Am Coll Cardiol 2010;55:1427-32.

18. Kahlert P, Knipp SC, Schlamann M, et al. Silent and apparent cerebral ischemia after percutaneous transfemoral aortic valve implantation: a diffusion-weighted magnetic resonance imaging study. Circulation 2010;121:870-8.

19. Segal AZ, Abernethy WB, Palacios IF, et al. Stroke as a complication of cardiac catheterization: risk factors and clinical features. Neurology 2001;56:975-7.

20. Amarenco P, Cohen A, Tzourio C, et al. Atherosclerotic disease of the aortic arch and the risk of ischemic stroke. N Engl J Med 1994;331:1474-9.

21. Di Tullio MR, Russo C, Jin Z, et al. Aortic arch plaques and risk of recurrent stroke and death. Circulation 2009;119:2376-82.

22. Meissner I, Khandheria BK, Sheps SG, et al. Atherosclerosis of the aorta: risk factor risk marker, or innocent bystander? A prospective population-based transesophageal echocardiography study. J Am Coll Cardiol 2004;44:1018-24.

23. Newman MF, Kirchner JL, Phillips-Bute B, et al. Longitudinal assessment of neurocognitive function after coronary-artery bypass surgery. $N$ Engl J Med 2001;344:395-402.

24. Knipp SC, Matatko N, Schlamann M, et al. Small ischemic brain lesions after cardiac valve replacement detected by diffusion-weighted magnetic resonance imaging: relation to neurocognitive function. Eur J Cardiothorac Surg 2005;28:88-96.

25. Yoshitake T, Kiyohara Y, Kato I, et al. Incidence and risk factors of vascular dementia and Alzheimer's disease in a defined elderly Japanese population: the Hisayama Study. Neurology 1995;45:1161-8.

26. Gurvitch R, Webb JG. Life after transcatheter aortic valve implantation: quality still matters. Heart 2010;96:1083-4.

27. Gotzmann $\mathbf{M}$, Hehen $T$, Germing $A$, et al. Short-term effects of transcatheter aortic valve implantation on neurohormonal activation, quality of life and 6-minute walk test in severe and symptomatic aortic stenosis. Heart 2010;96:1102-6.

28. Krane M, Deutsch MA, Bleiziffer $\mathbf{S}$, et al. Quality of life among patients undergoing transcatheter aortic valve implantation. Am Heart J 2010;160:451-7.

29. Ussia GP, Mulè M, Barbanti M, et al. Quality of life assessment after percutaneous aortic valve implantation. Eur Heart J 2009;30:1790-6.

30. Nietlispach F, Wijesinghe N, Gurvitch R, et al. An embolic deflection device for aortic valve interventions. JACC Cardiovasc Interv 2010:3:1133-8.

Heart alerts

Sign up for our electronic table of contents alert and you will never miss new issues of Heart when published online. Stay ahead and up to date by visiting heart.bmj.com. 